\title{
Tensor Products and Approximation Problems of $C^{*}$-Algebras ${ }^{1)}$
}

\author{
By
}

\author{
Jun TomiYama*
}

\begin{abstract}
For $C^{*}$-algebras $C$ and $D$ and their $C^{*}$-subalgebras $A$ and $B$, it is shown with other related results that if all irreducible representations of $A$ are finite dimensional with bounded degree then the family of product functionals in the minimal $C^{*}$-tensor product $C \underset{\alpha}{\otimes} D$ determines completely the subalgebra $A \underset{\alpha}{\otimes} B$. Relations to Effros' recent work ${ }^{\alpha}$ are also discussed.
\end{abstract}

\section{§1. Introduction}

Let $C$ and $D$ be $C^{*}$-algebras. In the case of the minimal tensor product of $C^{*}$-algebras, $C \underset{\alpha}{\otimes} D$, the family of product functionals determines completely the norm in $C \otimes D$. Nevertheless, once we are concerned with the structure of the algebra there appear many things in which we do not know the effect of the family of product functionals. Some of them give rise to basic difficulties in the theory of tensor products of $C^{*}$-algebras. For example, for a non-zero ideal $I$ of $C$ we still do not know in general whether the kernel of the homomorphism

$$
C \otimes \underset{\alpha}{\longrightarrow} D \longrightarrow I \underset{\alpha}{\otimes} D
$$

coincides with the tensor product $I \underset{\alpha}{\otimes D}$. In this case, one may easily see that the family of product functionals can not separate the kernel from $I \otimes D$.

In ${ }^{\alpha}$ the following we shall discuss such kind of the problem whether

Communicated by H. Araki, September 10, 1974.

* Faculty of Science, Yamagata University, Yamagata.

1) Work done during the author's stay as a Co-operative Research Member at the Research Institute for Mathematical Sciences, Kyoto University. 
the family of product functionals can determine a product subalgebra $A \otimes_{\alpha} B$, that is, whether to each element $x$ not belonging to $A \otimes_{\alpha} B$ there exists a product functional $\varphi \otimes \psi$ vanishing at $A \otimes B$ with $\langle x, \varphi \otimes \psi(\psi \neq 0$. The problem will be formulated in terms of a certain extraordinary product (Fubini product) of subalgebras $A$ and $B$ in $C \underset{\alpha}{\otimes} D$. If one recalls results in Grothendieck [8] or Waelbroeck [19] one may regard the problem as a $C^{*}$-algebraic version of the usual approximation problem for Banach spaces, which had been remained open for a long time until Enflo [6] gave the first counter-example.

We shall give in $\S 2$ a description of our problem as well as formulations of the problem. The main result will be stated in $\S 3$. In spite of the depth of the problem, to date all that we have succeeded in proving is that (Theorem 3.1) if $A$ is a $C^{*}$-algebra whose irreducible representations are finite dimensional with bounded degree, then the question is affirmative for any triple of $C^{*}$-algebras $(B, C, D)$. In $\S 4$ we shall show relations and difference between our Fubini product and an extraordinary product defined recently in Effros [4] as another $C^{*}$-algebraic analogue of the approximation problem of Banach spaces. Next $\$ 5$ concerns a little different subject from others. The result here says, roughly speaking, that for a generalized Fubini map defined in $\S 4$ the Fubini type principle (which will be shown to be quite useful throughout this paper) holds if and only if the induced functional is $\sigma$-weakly continuous.

The author would like to thank G. A. Elliott and M. Takesaki for their stimulating conversation on the subject and $\mathbb{H}$. Araki for his hospitality during the author's stay at the Research Institute for Mathematical Sciences, Kyoto University.

\section{§2. Formulations of the Problems}

Let $C$ and $D$ be $C^{*}$-algebras and $A$ and $B$ be $C^{*}$-subalgebras of $C$ and $D$ respectively. Throughout this paper we do not assume units for these $C^{*}$-algebras unless otherwise specified. When the algebras $C$ and $D$ are acting on Hilbert spaces $H$ and $K$ the minimal $C^{*}$-tensor product $\underset{\alpha}{\otimes} D$ is defined as the closure of the algebraic tensor product 
$C \otimes D$ by the operator norm of the algebra of all bounded operators on $H \otimes K$. Hence the product algebra $A \otimes B$ is considered as a $C^{*}$ subalgebra of $C \underset{\alpha}{\otimes}$. For each bounded linear functional $\varphi$ on $C$ (resp. $\psi$ on $D$ ) we can define a bounded linear mapping $R_{\varphi}$ from $C \otimes D$ to $D$ (resp. $L_{\psi}$ from $C \underset{\alpha}{\otimes} D$ to $C$ ) which we call a right Fubini map (resp. left Fubini map), such that

$$
\begin{aligned}
& R_{\varphi}\left(\sum_{i=1}^{n} a_{i} \otimes b_{i}\right)=\sum_{i=1}^{n}\left\langle a_{i}, \varphi\right\rangle b_{i}, \\
& L_{\psi}\left(\sum_{i=1}^{n} a_{i} \otimes b_{i}\right)=\sum_{i=1}^{n}\left\langle b_{i}, \psi\right\rangle a_{i} .
\end{aligned}
$$

These two kinds of mappings are related in the following way;

$$
\langle x, \varphi \otimes \psi\rangle=\left\langle R_{\varphi}(x), \psi\right\rangle=\left\langle L_{\psi}(x), \varphi\right\rangle .
$$

We call this the Fubini type principle since it may be regarded as a Fubini theorem for the product functional $\varphi \otimes \psi$. It is shown in [14], [16] that these mappings and the above equality are quite useful in the theory of the minimal tensor product of $C^{*}$-algebras. follows;

We define a closed subspace $F(A, B)$ with respect to $C \underset{\alpha}{\otimes} D$ as

$$
F(A, B)=\left\{x \in C \underset{\alpha}{\otimes} D ; R_{\varphi}(x) \in B, L_{\psi}(x) \in A \text { for every } \varphi \in C^{*} \text { and } \psi \in D^{*}\right\},
$$

where $C^{*}$ and $D^{*}$ mean the duals of the Banach spaces $C$ and $D$. We call $F(A, B)$ the Fubini product of $A$ and $B$ with respect to $C \underset{\alpha}{\otimes} D$. We have following natural questions.

Question 1. Does $F(A, B)$ necessarily coincide with $\underset{\alpha}{A \otimes B}$ ?

Question 2. Does $F(A, B)$ contain a non-trivial (not equal to $A \underset{\alpha}{\otimes} B$ ) $C^{*}$-subalgebra of $C \underset{\alpha}{\otimes} D$ ?

One may easily see that equivalent formulations of the above questions are;

Problem 1. For each element $x$ in $C \otimes D$ not belonging to $A \otimes B$, can we find a product functional $\varphi \otimes \psi^{\alpha}$ vanishing at $A \underset{\alpha}{\otimes} B$ with 
$\langle x, \varphi \otimes \psi\rangle \neq 0 ?$

Problem 2. For each $C^{*}$-subalgebra $E$ which contains $A \otimes B$ properly, can we find a product functional which vanishes on $A \otimes B$ and which does not vanish on $E$ ?

As we have noticed in the introduction many basic questions in the theory of $C^{*}$-tensor products appear in the form of these problems, particularly in the form of problem 2. To illustrate the thing, we add two more examples. Let $K_{1}, K_{2}$ and $K$ be the largest GCR ideals in $A, B$ and $A \otimes B$. Then most of the problems related to the type of the tensor product $A \otimes B$ are reduced to the question whether $K=K_{1} \otimes K_{2}$ or not. A partial answer to this question is known ([16; Theorem 4]) but in general all what we know is that $K$ is contained in the Fubini product $F\left(K_{1}, K_{2}\right)$ with respect to $A \underset{\alpha}{\otimes}$ (cf. [16; p 225]). The second one is a question for relative commutants in $C^{*}$-tensor products, that is, the question whether the relative commutant of $A \otimes B$ in $C \otimes \underset{\alpha}{\otimes}$ coincides with the $C^{*}$-tensor product of the relative commutants ${ }^{\alpha}$ of $A$ in $C$ and $B$ in $D$. Again in this case the former is contained in the Fubini product of the latter. The first question also relates to the question of separation of closed ideals in $A \underset{\alpha}{\otimes} B$ by means of the family of all product functions, a stronger version. A partial answer to this stronger version is also given in [16; Theorem 5]. The second question is, of course, a $C^{*}$-algebraic version of the fundamental commutation theorem of the tensor products of von Neumann algebras which had remained open for a long time until the Tomita-Takesaki theory appeared. In this connection, it should be noticed that in case of von Neumann tensor products we can define similarly a $\sigma$-weakly closed Fubini product for a given von Neumann tensor product by means of $\sigma$ weakly continuous Fubini mappings and we get an affirmative answer to Question 1. Namely, let $M$ and $N$ be von Neumann algebras acting on Hilbert spaces $H$ and $K$. Denote by $B(H)$ and $B(K)$ the algebras of all bounded linear operators on $H$ and $K$, respectively. For each $\sigma$-weakly continuous linear functional $\varphi$ on $B(H)$, i.e. $\varphi \in B(H)_{*}$, we can define a $\sigma$-weakly continuous right Fubini map $R_{\varphi}$ from the von Neumann tensor product $B(H) \bar{\otimes} B(K)$ to $B(K)$. Similarly a $\sigma$-weakly continuous linear functional $\psi$ on $B(K)$, i.e. $\psi \in B(K)_{*}$, gives rise a $\sigma$ - 
weakly continuous left Fubini map $L_{\psi}$ from $B(H) \bar{\otimes} B(K)$ to $B(H)$. Define the subspace $F(M, N)$ in $B(H) \bar{\otimes} B(K)$ by

$$
F(M, N)=\left\{x \in B(H) \bar{\otimes} B(K) ; R_{\varphi}(x) \in N, L_{\psi}(x) \in M\right.
$$

$$
\text { for every } \left.\varphi \in B(H)_{*}, \psi \in B(K)_{*}\right\} \text {. }
$$

Then it is nothing but an equivalent version of the commutation theorem $(M \bar{\otimes} N)^{\prime}=M^{\prime} \bar{\otimes} N^{\prime}$ that we can conclude $F(M, N)=M \bar{\otimes} N \quad$ (cf. $[17 ; \S 2])$. The result has many interesting consequences. For instance, for all von Neumann algebras $M_{1}$ and $M_{2}$ on $H$ and $N_{1}$ and $N_{2}$ on $K$, the intersection $M_{1} \bar{\otimes} N_{1} \cap M_{2} \bar{\otimes} N_{2}$ is contained in $F\left(M_{1} \cap M_{2}, N_{1}\right.$ $\cap N_{2}$ ), whence

$$
M_{1} \bar{\otimes} N_{1} \cap M_{2} \bar{\otimes} N_{2}=\left(M_{1} \cap M_{2}\right) \bar{\otimes}\left(N_{1} \cap N_{2}\right) .
$$

A particular case of this fact is the result for relative commutants; if $M_{1}$ and $N_{1}$ are von Neumann subalgebras of $M_{2}$ and $N_{2}$ then we have

$$
\left(M_{1} \bar{\otimes} N_{1}\right)^{\prime} \cap M_{2} \bar{\otimes} N_{2}=M_{1}^{\prime} \bar{\otimes} N_{1}^{\prime} \cap M_{2} \bar{\otimes} N_{2}=\left(M_{1}^{\prime} \cap M_{2}\right) \bar{\otimes}\left(N_{1}^{\prime} \cap N_{2}\right) .
$$

If $\mathscr{F}$ is a group of *-automorphisms of $M$ and if $\mathscr{G}$ is a group of $*$-automorphisms of $N$, then the subalgebra of fixed points for $\mathscr{F} \times \mathscr{G}$ acting as a group of *-automorphisms of $M \bar{\otimes} N$ is contained in $F\left(M_{\mathscr{F}}\right.$, $N_{\mathscr{G}}$ ) of the subalgebras of fixed points $M_{\mathscr{F}}$ and $N_{\mathscr{G}}$. Hence we have

$$
(M \bar{\otimes} N)_{\mathscr{F} \times \mathscr{G}}=M_{\mathscr{F}} \bar{\otimes} N_{\mathscr{G}} .
$$

In spite of these positive results the author is afraid that the answer to Question 1 for $C^{*}$-tensor products could be negative in general. We note two preliminary lemmas.

Lemma 2.1. Each element $x \in F(A, B)$ gives rise a linear mapping from $A^{*}$ to $B$ whose restriction to the unit sphere of $A^{*}$ is weak continuous. It also defines a linear mapping from $B^{*}$ to $A$ whose restriction to the unit sphere is weak* continuous.

Proof. Let $\omega$ be a bounded linear functional on $A$ and let $\varphi$ and $\phi$ be extensions of $\omega$ to $C$. Then for an arbitrary $\psi \in D^{*}$ we 
have

$$
\begin{aligned}
& \left\langle R_{\varphi}(x), \psi\right\rangle=\langle x, \varphi \otimes \psi\rangle=\left\langle L_{\psi}(x), \varphi\right\rangle \\
& \quad=\left\langle L_{\psi}(x), \omega\right\rangle=\left\langle L_{\psi}(x), \phi\right\rangle=\langle x, \phi \otimes \psi\rangle \\
& =\left\langle R_{\phi}(x), \psi\right\rangle .
\end{aligned}
$$

Hence the image $R_{\varphi}(x)$ in $B$ does not depend on the choice of the extensions of $\omega$. We denote this image by $R(\omega, x)$. The mapping $R(\omega, x)$ is apparently a linear mapping from $A^{*}$ to $B$. On the other hand, one easily verifies that the mapping $R_{\varphi}(x)$ from $C^{*}$ to $D$ is weak* continuous on the unit sphere of $C^{*}$. Hence if we consider norm preserving extensions of elements in the unit sphere of $A^{*}$ to $C^{*}$ and the weak* $^{*}$ compactness of the unit sphere of $C^{*}$ we may conclude that $R(\omega$, $x)$ is weak* continuous on the unit sphere of $A^{*}$.

Lemma 2.2. Let $I$ and $J$ be closed ideals of $A$ and $B$, respectively. Then the Fubini product $F(I, J)$ with respect to $A \otimes B$ is a closed ideal.

Proof. Take a fixed $x \in F(I, J)$. It is enough to show that both $(a \otimes b) x$ and $x(a \otimes b)$ belong to $F(I, J)$ for $a \in A$ and $b \in B$. Take $\varphi \in A^{*}$ and $\psi \in B^{*}$. We denote by $L_{a} \varphi$ the functional defined by

$$
\left\langle y, L_{a} \varphi\right\rangle=\langle a y, \psi\rangle
$$

Then,

$$
\begin{aligned}
& \left\langle R_{\varphi}((a \otimes b) x), \psi\right\rangle=\langle(a \otimes b) x, \varphi \otimes \psi\rangle \\
& \quad=\left\langle x, L_{a} \varphi \otimes L_{b} \psi\right\rangle=\left\langle R_{L_{a} \varphi}(x), L_{b} \psi\right\rangle=\left\langle b R_{L_{a} \varphi}(x), \psi\right\rangle .
\end{aligned}
$$

Hence we have

$$
R_{\varphi}((a \otimes b) x)=b R_{L_{a} \varphi}(x) \in J .
$$

Similarly we see that

$$
L_{\psi}((a \otimes b) x)=a L_{L_{b} \psi}(x) \in I .
$$

Thus, $(a \otimes b) x \in F(I, J)$. A similar argument shows that $x(a \otimes b) \in F(I, J)$. 
Next we shall describe a relation of our problem to the usual approximation problem of Banach spaces. Let $E_{1}$ and $E_{2}$ be Banach spaces and denote by $E_{1} \widehat{\widehat{\otimes}} E_{2}$ the injective tensor product of $E_{1}$ and $E_{2}$. Waelbroech [17] defined a product $E_{1} \varphi E_{2}$ as one of the following three mutually isometric Banach spaces:

a) The space of all linear mappings of $E_{1}^{*}$ into $E_{2}$ whose restriction to the unit sphere of $E_{1}^{*}$ are weak* continuous.

b) The space of all linear mappings of $E_{2}^{*}$ into $E_{1}$ whose restriction to the unit sphere of $E_{2}^{*}$ are weak* continuous.

c) The space of all bilinear forms on $E_{1}^{*} \times E_{2}^{*}$ whose restriction to the product of the unit sphere are weak* continuous.

A Banach space $E$ is said to have the approximation property if each compact operator on $E$ is a uniform limit of finite rank operators. Then a result in [19] says that $E_{1} \widehat{\widehat{\otimes}} E_{2}$ coincides with $E_{1} \varphi E_{2}$ for every Banach space $E_{2}$ if and only if $E_{1}$ satisfies the approximation property. Since we now have a counter-example to the approximation problem constructed by Enflo [6], we know that there is a case such that $E_{1}$ $\widehat{\otimes} E_{2} \subsetneq E_{1} \varphi E_{2}$. Now let $X$ and $Y$ be the unit sphere of $E_{1}^{*}$ and $E_{2}^{*}$ with weak ${ }^{*}$ topology. The space $E_{1}$ (resp. $E_{2}$ ) can be considered as a closed subspace of $C(X)$ (resp. $C(Y)$ ), the space of complex valued continuous functions on $X$ (resp. on $Y$ ). Moreover the space $E_{1} \widehat{\widehat{\otimes}} E_{2}$ can be considered as a subspace of $C(X) \widehat{\otimes} C(Y)$, which by $[8 ; \mathrm{p} 90]$, may be regarded as the space of $C(Y)$-valued continuous functions on $X$ or the space of $C(X)$-valued continuous functions on $Y$. Since in this case we can also define right and left Fubini mappings for $\varphi \in C(X)^{*}$ and $\psi \in C(Y)^{*}$, we may define the Fubini product $F\left(E_{1}, E_{2}\right)$ with respect to $C(X) \widehat{\otimes} C(Y)$, too. Then it is not so hard to see that

$$
F\left(E_{1}, E_{2}\right)=C(X) \widehat{\widehat{\otimes}} E_{2} \cap E_{1} \widehat{\widehat{\otimes}} C(Y)=C\left(X, E_{2}\right) \cap C\left(Y, E_{1}\right),
$$

which shows that the space $F\left(E_{1}, E_{2}\right)$ is just another definition of Waelbroeck's product $E_{1} \varphi E_{2}$. Thus, in this case we have a nice characterization of the Fubini product, especially because of the simple definition of the injective cross-norm. At any rate, the above arguments show that our problem may be regarded as an approximation problem for $C^{*}$-algebras. We note at last that in spite of our Lemma 2.1 the 
Fubini product is not isometric to a space of linear mappings as in Waelbroeck's definition except for a few cases.

\section{§3. Main Theorem}

In this section we shall prove the following

Theorem 3.1. Let $C$ and $D$ be $C^{*}$-algebras and $A$ and $B$ be their $C^{*}$-subalgebras, respectively. If $A$ is a $C^{*}$-algebra whose irreducible representations are finite dimensional with bounded degree, then Question 1 has an affirmative answer, that is, $F(A, B)=A \underset{\alpha}{\otimes} B$.

The proof will be decomposed into a sequence of lemmas.

Before going into the proof we list some consequences of the above result.

Corollary 1. Let $I$ be a closed ideal of $C$ with the property mentioned above. Then the kernel of the homomorphism

$$
C \underset{\alpha}{\otimes} D \longrightarrow C / I \underset{\alpha}{\otimes} D \text { is } I \underset{\alpha}{I \otimes} D
$$

The result is previously known with restrictions to the algebra $C$ (such as $C$ is of type $I$ etc.).

Corollary 2. If one of the relative commutants of $A$ and $B$ in $C$ and $D$ is a $C^{*}$-algebra in the class mentioned above, in particular a commutative $C^{*}$-algebra, then

$$
(A \underset{\alpha}{\otimes} B)^{\prime} \cap C \underset{\alpha}{\otimes} D=\left(A^{\prime} \cap C\right) \underset{\alpha}{\otimes}\left(B^{\prime} \cap D\right)
$$

Lemma 3.2. If $A$ is the algebra of $n \times n$-matrices, then Question 1 has an affirmative answer.

Proof. Take a fixed $x \in F(A, B)$. There exists a projection of norm one $\varepsilon$ from $C$ to $A$. Take $\varphi \in C^{*}$ and $\psi \in D^{*}$ and denote by $\varphi \mid A$ the restriction of $\varphi$ to $A$. Then,

$$
\langle\varepsilon \otimes 1(x), \quad \varphi \otimes \psi\rangle=\left\langle x,{ }^{t} \varepsilon(\varphi \mid A) \otimes \psi\right\rangle=\langle x, \varphi \otimes \psi\rangle .
$$


Hence $x=\varepsilon \otimes 1(x) \in A \otimes D$. Let $\left(x_{i j}\right)$ be a matrix representation of $x$ over $D$. For each $\psi^{\alpha} \in D^{*}$, one easily verifies that $L_{\psi}(x)=\left(\psi\left(x_{i j}\right)\right)$. If $\psi$ vanishes on $B$, We have, for each $\varphi \in A^{*}$,

$$
\left\langle L_{\psi}(x), \varphi\right\rangle=\left\langle R_{\varphi}(x), \psi\right\rangle=0 .
$$

Hence,

$$
L_{\psi}(x)=\left(\psi\left(x_{i j}\right)\right)=0 \quad \text { and } \quad \psi\left(x_{i j}\right)=0 \quad \text { for every } i \text { and } j .
$$

Therefore, each entry $x_{i j}$ belongs to $B$, which shows that $x$ belongs to $A \otimes B$.

Let $A$ be an $n$-homogeneous $C^{*}$-algebra, which means that every irreducible representation of $A$ is $n$-dimensional. Put $X=\hat{A}$, the dual of $A$. We write $P_{t}$ for the primitive ideal corresponding to the representation $t \in X$. Denote by $A(t)$ the image of $A$ by $t$. The image of an element $a$ will be denoted by $a(t)$. In this case the space $X$ is a locally compact Hausdorff space and the function $t \rightarrow\|a(t)\|$ is continuous and vanishes at infinity. Let $\mathscr{F}$ be the set of all operator fields in $\{X, A(t)\}$ induced by the elements of $A$. A field $x(t)$ in $\{X, A(t)\}$ is said to be continuous with respect to $\mathscr{F}$ if for any $t \in X$ and positive $\varepsilon$ there is a field $a$ in $\mathscr{F}$ and a neighborhood $U$ of $t$ such that

$$
\|x(s)-a(s)\|<\varepsilon \quad \text { for every } \quad s \in U .
$$

It is known that the algebra $A$ is represented as the $C^{*}$-algebra of all continuous fields in $\{X, A(t), \mathscr{F}\}$ vanishing at infinity with local triviality ([7], [11]). Now, consider the homomorphism $t \otimes 1$ of $A \otimes B$. The kernel of this homomorphism is $P_{t} \otimes_{\alpha} B$ by $[14 ;$ Theorem 5]. Each element $x \in A \otimes_{\alpha} B$ gives rise to an operator field $x(t)=t \otimes 1(x)$ in $\{X, A(t) \underset{\alpha}{\otimes} B\}$. We have

Lemma 3.3. The function $t \rightarrow\|x(t)\|$ is continuous on $X$ and vanishes at infinity. The algebra $A \underset{\alpha}{\otimes} B$ is isomorphic to the algebra of continuous fields in $\{X, A(t) \underset{\alpha}{\otimes} B\}^{\alpha}$ with respect to the fields $x(t)$ 's.

Proof. Fix $t \in X$. By the local triviality there exists a compact neighborhood $U$ of $t$, on which the restriction of $A$ may be regarded 
as the space of $M_{n}$-valued continuous functions. Since the latter space is isomorphic to the tensor product $C(U) \underset{\alpha}{\otimes} M_{n}$, the restriction of the space of the fields $x(t)$ 's to $U$ may be naturally identified with $C(U)$ $\underset{\alpha}{\otimes} M_{n} \underset{\alpha}{\otimes}$. Thus the function $s \rightarrow\|x(s)\|$ is continuous on $U$, in particular at $t$. Moreover if a net $\left\{t_{\alpha}\right\}$ is eventually outside any compact set, then $\left\|a\left(t_{\alpha}\right)\right\|$ converges to zero for every $a \in A$. Hence the function $\left\|\sum_{i=1}^{n} a_{i}\left(t_{\alpha}\right) \otimes b_{i}\right\|$ converges to zero. Therefore the function $\|x(t)\|$ vanishes at infinity, too.

Next suppose $x(t)=0$ for every $t \in X$. Then for every $\psi \in B^{*}$, $L_{\psi}(x)$ belongs to the ideal $P_{t}$ for every $t$. Hence $L_{\psi}(x)=0$ for every $\psi \in B^{*}$, which implies $x=0$. Thus we have an isomorphism of $A \underset{\alpha}{\otimes} B$ into the space of continuous fields. We assert this is an onto mapping. Consider an arbitrary couple $(t, s)$ in $X$. We have

$$
\left.\overline{P_{t} \otimes} \underset{\alpha}{\otimes} B+P_{s} \underset{\alpha}{\otimes B} \supseteq \overline{\left(P_{t}+P_{s}\right.}\right) \underset{\alpha}{\otimes} B=A \underset{\alpha}{\otimes} B
$$

Hence by [9; Lemma 8.1]

$$
P_{t} \otimes_{\alpha} B+P_{s} \underset{\alpha}{\otimes} B=A \underset{\alpha}{\otimes} B
$$

Let $a$ and $b$ be arbitrary elements of $A(t) \otimes B$ and $A(s) \otimes B$, respectively. Choose $c$ and $d$ in $A \underset{\alpha}{\otimes} B$ with $t \otimes 1(c)=\stackrel{\alpha}{a}$ and $s \otimes 1(d)=b$. We may write

$$
c-d=x-y
$$

with $x \in P_{t} \underset{\alpha}{\otimes} B$ and $y \in P_{s} \underset{x}{\otimes B}$. Put

$$
z=c-x=d-y .
$$

Then we have

$$
a=t \otimes 1(z) \quad \text { and } \quad b=s \otimes 1(z)
$$

Hence by the general Stone-Weierstrass theorem for $C^{*}$-algebras of continuous fields [15; Theorem 2.2], the assertion follows. This completes the proof. 
Remark. The above proof shows that the result is valid for any $C^{*}$-algebra of continuous fields with local triviality.

Now we are back to our original situation assuming that $A$ is $n$-homogeneous. We extend the representation $t$ to a representation $\pi_{t}$ of $C$ on a space $K$ such that the restriction of $\pi_{t}(A)$ to a subspace $H$ is unitarily equivalent to $t$ (cf. $[3 ; 2.10 .2]$ ). Let $p$ be the projection onto $H$ and $z(t)$ be the central cover of $p$ in $\pi_{t}(A)^{\prime \prime}$, the commutant of $\pi_{t}(A)^{\prime}$.

We assume that the algebras $B$ and $D$ are acting on a Hilbert space.

Lemma 3.4. Suppose $x \in F(A, B)$. Then

$$
\pi_{t} \otimes 1(x) z(t) \otimes 1 \in \pi_{t}(A) z(t) \underset{\alpha}{\otimes} B
$$

Proof. We shall show that the element $\pi_{t} \otimes 1(x) z(t) \otimes 1$ belongs to $F\left(\pi_{t}(A) z(t), B\right)$ with respect to $\pi_{t}(C)^{\prime \prime} \otimes D$. Then, as $\pi_{t}(A) z(t)$ is isomorphic to $M_{n}$ the conclusion follows from Lemma 3.2.

Now for every $\varphi \in\left(\pi_{t}(C)^{\prime \prime}\right)^{*}$ and $\psi \in D^{*}$, we have

$$
\begin{aligned}
& \left\langle R_{\varphi}\left(\pi_{t} \otimes 1(x) z(t) \otimes 1\right), \psi\right\rangle=\left\langle\pi_{t} \otimes 1(x), R_{z(t)} \varphi \otimes \psi\right\rangle \\
& \quad=\left\langle x, R_{z(t)} \varphi \circ \pi_{t} \otimes \psi\right\rangle=\left\langle R_{R_{z(t)} \varphi \circ \pi_{t}}(x), \psi\right\rangle .
\end{aligned}
$$

Whence

$$
R_{\varphi}\left(\pi_{t} \otimes 1(x) z(t) \otimes 1\right)=R_{R_{z(t) \varphi \circ \pi_{t}}}(x) \in B
$$

Similarly we get

$$
L_{\psi}\left(\pi_{t} \otimes 1(x) z(t) \otimes 1\right)=\pi_{t}\left(L_{\psi}(x)\right) z(t) \in \pi_{t}(A) z(t) .
$$

Remark. The element $\pi_{t} \otimes 1(x)$ commutes with $z(t) \otimes 1$. In fact,

$$
\begin{aligned}
& \left\langle z(t) \otimes 1 \pi_{t} \otimes 1(x), \varphi \otimes \psi\right\rangle=\left\langle x, L_{z(t)} \varphi \circ \pi_{t} \otimes \psi\right\rangle \\
& =\left\langle z(t) \pi_{t}\left(L_{\psi}(x)\right), \varphi\right\rangle=\left\langle\pi_{t}\left(L_{\psi}(x)\right) z(t), \varphi\right\rangle \\
& =\left\langle\pi_{t} \otimes 1(x) z(t) \otimes 1, \varphi \otimes \psi .\right\rangle
\end{aligned}
$$

Let $\theta_{t}$ be the isomorphism between $\pi_{t}(A) z(t)$ and $A(t)$ and denote $\theta_{t} \otimes 1$ 
the isomorphism of $\pi_{t}(A) z(t) \otimes B$ to $A(t) \otimes B$. By the above lemma there exists an element $x(t)$ in $A(t) \underset{\alpha}{\otimes} B$ with $\theta_{t} \otimes 1\left(\pi_{t} \otimes 1(x) z(t) \otimes 1\right)=x(t)$.

Lemma 3.5. The field $t \rightarrow x(t)$ is a continuous field in $\{X, A(t) \underset{\alpha}{\otimes} B\}$ vanishing at infinity.

Proof. Take a point $t \in X$ and let $U$ be a compact neighborhood of $t$ on which the algebra $A$ (or $A \underset{\alpha}{\otimes} B$ ) becomes trivial with complete matrix units $e_{i j}(s)$ for $s \in U$. Let $\varphi_{i j}^{\alpha}(s)$ be a linear functional on $A(s)$ with norm one such that

$$
\left\langle e_{k e}(s), \varphi_{i j}(s)\right\rangle=\delta_{k}^{i} \delta_{e}^{j}
$$

Let

$$
x(s)=\sum_{i, j=1}^{n} e_{i j}(s) \otimes x_{i j}(s) \quad \text { with } \quad x_{i j}(s) \in B
$$

Then for every $\psi \in D^{*}$ we get

$$
\begin{aligned}
& \left\langle x_{i j}(s), \psi\right\rangle=\left\langle x(s), \varphi_{i j}(s) \otimes \psi\right\rangle \\
& =\left\langle\pi_{s} \otimes 1(x) z(s) \otimes 1, \varphi_{i j}(s) \circ \theta_{s} \otimes \psi\right\rangle \\
& =\left\langle x, \phi_{i j}(s) \circ \pi_{s} \otimes \psi\right\rangle=\left\langle R_{\phi_{i j}(s) \circ \pi_{s}}(x), \psi\right\rangle
\end{aligned}
$$

where $\phi_{i j}(s)$ is a norm preserving extension of $R_{z(s)} \varphi_{i j}(s) \circ \theta_{s}$ to $\pi_{s}(C)$ whose choice does not change the value $R_{\phi_{i j}(s) \circ \pi_{s}}(x)$ because $x$ belongs to $F(A, B)$. It follows that $x_{i j}(s)=R_{\phi_{i j}(s) \circ \pi_{s}}(x)$. On the other hand, for $a \in A$, we have

$$
\begin{aligned}
& \left\langle a,{ }_{\phi i j}(s) \circ \pi_{s}\right\rangle=\left\langle\pi_{s}(a) z(s), \varphi_{i j}(s) \circ \theta_{s}\right\rangle \\
& =\left\langle a(s), \varphi_{i j}(s)\right\rangle=\lambda_{i j}(s)
\end{aligned}
$$

where $a(s)=\sum_{i, j=1}^{n} \lambda_{i j}(s) e_{i j}(s)$. Therefore if $s_{\alpha}$ converges to $s$ in $U, \phi_{i j}\left(s_{\alpha}\right)$ 。 $\pi_{s_{\alpha}} \mid A$ converges weakly to $\phi_{i j}(s) \circ \pi_{s} \mid A$. Now suppose $x(t)=y(t)$ for some $y \in A \underset{\alpha}{\otimes} B$. Then $x_{i j}(t)=y_{i j}(t)$. From the above arguments,

$$
x_{i j}\left(s_{\alpha}\right)-y_{i j}\left(s_{\alpha}\right)=R_{\phi_{i j}\left(s_{\alpha}\right) \circ \pi_{s \alpha}}(x-y),
$$


which converges to $R_{\phi_{i}(t) \circ \pi_{t}}(x-y)=0$ by Lemma 2.1 if $s_{\alpha}$ converges to $t$ in $U$. Therefore, $x(t)$ is a continuous field. Finally suppose $t_{\alpha}$ converges to infinity. We choose at each point $t_{\alpha}$ the functional $\varphi_{i j}\left(t_{\alpha}\right)$ considering a fixed neighborhood of $t_{\alpha}$ on which $A$ becomes trivial. Let $\left\{\psi_{i j}\left(t_{\alpha}\right)\right\}$ be norm preserving extensions of $R_{z\left(t_{\alpha}\right)} \varphi_{i j}\left(t_{\alpha}\right) \circ \theta_{t_{\alpha}}$ to $\pi_{t_{\alpha}}(C)$. Then there exists a weak* convergent subnet of $\left\{\psi_{\iota j}\left(t_{\alpha}\right) \circ \pi_{t_{\alpha}}\right\}$. Since this holds for each net $\left\{t_{\alpha}\right\}$, in order to get the conclusion we may suppose that the net $\left\{\psi_{i j}\left(t_{\alpha}\right) \circ \pi_{t_{\alpha}}\right\}$ itself converges weakly to a functional $\varphi_{0}$ in the weak* topology. As an element $a$ of $A$ vanishes at infinity in $X$ we have

$$
\begin{aligned}
& \left|\left\langle a, \varphi_{0}\right\rangle\right|=\lim _{\alpha}\left|\left\langle a, \psi_{i j}\left(t_{\alpha}\right) \circ \pi_{t_{\alpha}}\right\rangle\right|=\lim _{\alpha}\left|\left\langle a\left(t_{\alpha}\right), \varphi_{i j}\left(t_{\alpha}\right)\right\rangle\right| \\
& \leqq \lim _{\alpha}\left\|a\left(t_{\alpha}\right)\right\|=0 .
\end{aligned}
$$

Hence $\varphi_{0} \mid A=0$, which implies, by Lemma 2.1, that

$$
x_{i j}\left(t_{\alpha}\right)=R_{\psi_{i J}\left(t_{\alpha}\right) \circ \pi_{t_{\alpha}}}(x) \longrightarrow 0 .
$$

Therefore the field $x(t)$ vanishes at infinity.

Lemma 3.6. If $A$ is n-homogeneous, Question 1 has an affirmative answer.

Proof. Take an element $x \in F(A, B)$. By Lemma 3.5 and 3.3 there exists an element $y$ of $\underset{\alpha}{\otimes} B$ such that $x(t)=y(t)$ for every $t \in X$. This means that

$$
\pi_{t} \otimes 1(x-y) z(t) \otimes 1=0 \quad \text { for every } \quad t \in X
$$

Hence for every $\varphi \in\left(\pi_{t}(C)^{\prime \prime}\right)^{*}$ and $\psi \in D^{*}$, we have

$$
\begin{aligned}
& \left\langle\pi_{t} \otimes 1(x-y) z(t) \otimes 1, \varphi \otimes \psi\right\rangle=\left\langle x-y, R_{z(t)} \varphi \cdot \pi_{t} \otimes \psi\right\rangle \\
& =\left\langle L_{\psi}(x-y), R_{z(t)} \varphi \circ \pi_{t}\right\rangle=\left\langle\pi_{t}\left(L_{\psi}(x-y)\right) z(t), \varphi\right\rangle=0 .
\end{aligned}
$$

Hence $\pi_{t}\left(L_{\psi}(x-y)\right) z(t)=0 \quad$ and $\quad L_{\psi}(x-y)(t)=0$ because $L_{\psi}(x-y) \in A$. Hence $L_{\psi}(x-y)=0$ for every $\psi \in D^{*}$ and $x-y=0$. Thus $x$ belongs to $A \otimes \otimes_{\alpha} B$. 
Proof of the Theorem. Let $n_{1}<n_{2}<\cdots<n_{k}$ be the dimensions of irreducible representations of $A$ and let $I_{1}$ be the intersection of the kernels of irreducible representations of $A$ with dimension $n_{1}$. Let $\pi$ be a representation of $A$ on a Hilbert space with kernel $I_{1}$. Write $\hat{\pi}$ as the extension of $\pi$ to $C$ on a Hilbert space $K$ such that the restriction of $\hat{\pi}(A)$ to a suitable subspace $H$ of $K$ is unitarily equivalent to $\pi$. Let $z$ be the central cover of the projection $p: k \rightarrow H$ in $\hat{\pi}(A)^{\prime}$. Take an element $x \in F(A, B)$. As in the proof of Lemma 3.3 we can show that $\hat{\pi} \otimes 1(x) z \otimes 1$ belongs to $F(\hat{\pi}(A) z, B)$ with respect to $\hat{\pi}(C)^{\prime \prime} \otimes_{\alpha} D$. Since the set $\left\{t \in \hat{A}: \operatorname{dim} t=n_{1}\right\}$ is closed in $\hat{A}, \hat{\pi}(A) z$ is an $n$-homogeneous $C^{*}$-algebra. Hence by Lemma 3.6 we have $\hat{\pi} \otimes 1(x) z \otimes 1 \in \hat{\pi}(A) z \otimes B$. Let $y_{1}$ be an element of $A \otimes B$ such that $\hat{\pi} \otimes 1(x) z \otimes 1=\hat{\pi} \otimes 1\left(y_{1}\right) z \otimes 1$. We assert that $x-y_{1} \in F\left(I_{1}, \stackrel{a}{B}\right)$ with respect to $C \otimes D$. In fact, suppose there exist a bounded linear functional $\psi \in \underset{\alpha}{*}$ with $L_{\psi}\left(x-y_{1}\right) \notin I_{1}$. Then there exists a functional $\varphi \in \pi(A)^{*}$ such that

$$
\left\langle L_{\psi}\left(x-y_{1}\right), \varphi \circ \pi\right\rangle \neq 0 .
$$

Let $\theta$ be the isomorphism of $\hat{\pi}(A) z$ to $\pi(A)$, then

$$
\left\langle\hat{\pi}\left(L_{\psi}\left(x-y_{1}\right)\right) z, \varphi \circ \theta\right\rangle=\left\langle L_{\psi}\left(x-y_{1}\right), \varphi \circ \pi\right\rangle \neq 0 \text {. }
$$

However,

$$
\begin{aligned}
& \left\langle\hat{\pi}\left(L_{\psi}\left(x-y_{1}\right)\right) z, \varphi \circ \theta\right\rangle=\left\langle L_{\psi}\left(x-y_{1}\right), R_{z}(\varphi \circ \theta) \circ \hat{\pi}\right\rangle \\
& \quad=\left\langle x-y_{1}, R_{z}(\varphi \circ \theta) \pi \otimes \psi\right\rangle \\
& \quad=\left\langle\hat{\pi} \otimes 1\left(x-y_{1}\right) z \otimes 1, \varphi \circ \theta \otimes \psi\right\rangle=0 .
\end{aligned}
$$

This is a contradiction. Therefore, $L_{\psi}\left(x-y_{1}\right) \in I_{1}$ for every $\psi \in D^{*}$. On the other hand, as both $x$ and $y_{1}$ belong to $F(A, B)$ it is apparent that $R_{\varphi}\left(x-y_{1}\right) \in B$ for every $\varphi \in C^{*}$.

Let $I_{2}$ be a closed ideal of $A$ which is the intersection of irreducible representations with dimensions $n_{1}$ or $n_{2}$. Then the quotient algebra $I_{1} / I_{2}$ is $n_{2}$-homogeneous. Hence applying the same argument for $x$ $-y_{1} \in F\left(I_{1}, B\right)$ we can find an element $y_{2} \in I_{1} \underset{\alpha}{\otimes B}$ such that

$$
x-y_{1}-y_{2} \in F\left(I_{2}, B\right) \text {. }
$$


Continuing this process we can find $\left\{y_{1}, y_{2}, \ldots y_{k-2}\right\}$ with $y_{i} \in I_{i-1} \underset{\alpha}{\otimes} B$ such that

$$
x-y_{1}-y_{2}-\cdots-y_{k-2} \in F\left(I_{k-1}, B\right) \text {. }
$$

However the ideal $I_{k-1}$ is an $n_{k}$-homogeneous $C^{*}$-algebra, hence by Lemma 3.6

$$
x-y_{1}-y_{2}-\cdots-y_{k-2} \in I_{k-1} \underset{\alpha}{\otimes} B
$$

Therefore $x \in A \underset{\alpha}{\otimes} B$, which completes the proof.

There is one easy case which is not included in the above arguments.

Proposition 3.7. Keep the same notations as above. Suppose there exist projections of norm one $\varepsilon_{1}$ and $\varepsilon_{2}$ from $C$ to $A$ and $D$ to $B$. Then $F(A, B)=A \otimes B$.

Proof. By [14; Theorem 1] there is a projection $\varepsilon_{1} \otimes \varepsilon_{2}$ from $C \underset{\alpha}{\otimes} D$ to $A \otimes B$ such that

$$
\varepsilon_{1} \otimes \varepsilon_{2}\left(\sum_{i=1}^{n} a_{i} \otimes b_{i}\right)=\sum_{i=1}^{n} \varepsilon_{1}\left(a_{i}\right) \otimes \varepsilon_{2}\left(b_{i}\right) .
$$

Take an element $x$ in $F(A, B)$. Then for every $\varphi \in C^{*}$ and $\psi \in D^{*}$ we have

$$
\begin{aligned}
\left\langle\varepsilon_{1} \otimes \varepsilon_{2}(x), \varphi \otimes \psi\right\rangle & =\left\langle x,(\varphi \mid A) \circ \varepsilon_{1} \otimes(\psi \mid B) \circ \varepsilon_{2}\right\rangle \\
& =\langle x, \varphi \otimes \psi\rangle .
\end{aligned}
$$

The second equality follows from the property of $x$. Thus $x=\varepsilon_{1} \otimes \varepsilon_{2}(x)$ $\in A \otimes \otimes_{\alpha} B$.

An interesting class of $C^{*}$-algebras satisfying the above condition is the class of injective von Neumann algebras. A von Neumann algebra $M$ acting on a Hilbert space $H$ is said to be injective if there is a projection of norm one from $B(H)$ to $M$ (we prefer the terminology 
"injective" in Effros and Lance [5] to our original terminology, a $C^{*}$-algebra having the extension property in $\left.[17 ; \S 7]\right)$. It turns out ([17; Theorem 7.2]) that the property is equivalent to saying that there is a projection of norm one to $M$ from any $C^{*}$-algebra containing $M$. Every von Neumann algebra of type $I$ and every hyperfinite factor together with its commutant are injective.

For von Neumann algebras there is an equivalent question for the Fubini product. Namely, let $M$ and $N$ be von Neumann algebras on Hilbert spaces $H$ and $K$. Then does the intersection $M \bar{\otimes} N \cap B(H)$ $\underset{\alpha}{\otimes} B(K)$ coincide with $M \underset{\alpha}{\otimes}$ ? The situation is expressed in the following way.

Proposition 3.8. $M \bar{\otimes} N \cap B(H) \underset{\alpha}{\otimes} B(K)$ coincides with $F(M, N)$ with respect to $B(H) \underset{\alpha}{\otimes} B(K)$. In particular, if $M$ and $N$ are injective we have

$$
\overline{M \otimes} N \cap B(H) \underset{\alpha}{\otimes} B(K)=M \underset{\alpha}{\otimes} N
$$

Proof. From the definition it is apparent that for any $\varphi \in B(H)_{*}$ $R_{\varphi}$ maps $M \bar{\otimes} N \cap B(H) \underset{\alpha}{\otimes} B(K)$ to $N$. On the other hand, an element $x \in B(H) \otimes B(K)$ gives rise to a linear mapping $R_{\varphi}(X)$ from $B(H)^{*}$ to $B(K)$ whose restriction to the unit sphere is weak* continuous. Since the unit sphere of the predual $B(H)_{*}$ is weak $^{*}$ dense in the unit sphere of $B(H)^{*}$, it follows that

$$
R_{\varphi}(\overline{M \otimes} N \cap B(H) \underset{\alpha}{\otimes} B(K))=N \quad \text { for every } \quad \varphi \in B(H)^{*} .
$$

Similarly we can see that

$$
L_{\psi}(M \bar{\otimes} N \cap B(H) \underset{\alpha}{\otimes} B(K))=M \quad \text { for every } \psi \in B(K)^{*} .
$$

The other inclusion follows from the commutation theorem for von Neumann tensor products as explained in $\S 2$. The rest is then just an immediate consequence of Proposition 3.7. 


\section{§4. Relations between the Fubini Products and the Extraordinary Products by Effros}

Recently Effros [4] has introduced an extraordinary product of two unital $C^{*}$-algebras as a $C^{*}$-algebraic analogue of the approximation property of Banach spaces. The definition goes as follows. Let $A$ and $B$ be $C^{*}$-algebras with units. Let $A^{* *}$ and $B^{* *}$ be the second duals of $A$ and $B$ as the enveloping von Neumann algebras for $A$ and $B$ respectively. Let $S_{*}$ be the set of normal states of $A^{* *} \bar{\otimes} B^{* *}$. Since the predual of $A^{* *} \bar{\otimes} B^{* *}$ is the tensor product $A^{*} \otimes B^{*}$ with the functional cross norm $\alpha^{\prime}, S_{*}$ is a subset of $A^{*} \underset{\alpha^{\prime}}{\otimes} B^{*}$. We provide it with the weak topology defined by $A \otimes B$ and consider this as a replacement of the product space of the unit spheres of the dual spaces in the case of the injective tensor products. Then, as in the case of Banach spaces, a $C^{*}$-algebra $D$ with

$$
A \underset{\alpha}{\otimes} B \subset D \subset A^{* *} \bar{\otimes} B^{* *}
$$

is said to be an extraordinary product of $A$ and $B$ provided the first inclusion is proper and the elements of $D$ are continuous on $S_{*}$ in the $A \otimes B$-topology. Since an element of $D$ may sit outside the $C^{*}$ tensor product $A^{* *} \underset{\alpha}{\otimes} B^{* *}$, this product may certainly be different from our Fubini product. However we can define a slightly weaker Fubini product;

$$
F_{\omega}(A, B)=\left\{x \in A^{* *} \bar{\otimes} B^{* *} ; R_{\varphi}(x) \in B, L_{\psi}(x) \in A \text { for every } \varphi \in A^{*} \text { and } \psi \in B^{*}\right\} .
$$

Then one can verify easily that $F_{\omega}(A, B)$ contains the algebra $D$. However, the following example shows that even in the case of commutative $C^{*}$-algebras this weak Fubini product may contain a $C^{*}$-subalgebra which does not coincide with $A \underset{\alpha}{\otimes}$, whereas in commutative cases the algebra does not have an extraordinary product by [4; Theorem 4.1] (or use the fact $\alpha$-norm=injective cross norm). Before going to state the example we need a little more preparation. A positive functional $\varphi$ on a von Neumann algebra $M$ is said to be singular if for every non-zero projection $p$ there is a non-zero projection $q \leqq p$ such that $\langle q, \varphi\rangle=0$. 
The set of finite linear combinations of singular positive linear functional forms a closed subspace of $M^{*}, M_{*}^{\perp}$. The conjugate space $M^{*}$ is decomposed into the $l_{1}$-sum of the predual $M_{*}$ and $M_{*}^{\perp}$, that is,

$$
\varphi=\varphi_{1}+\varphi_{2} \quad \text { with } \quad \varphi_{1} \in M_{*}, \quad \varphi_{2} \in M_{*}^{\perp} \quad \text { and } \quad\|\varphi\|=\left\|\varphi_{1}\right\|+\left\|\varphi_{2}\right\|
$$

(cf. [12]).

We call a functional in $M_{*}^{\frac{1}{*}}$ singular and a functional in $M_{*}$ normal.

Example. Put $A=B=l^{\infty}$. Let $e_{n}$ be the minimal projection of $l^{\infty}$ coresponding to the $n$-th coordinate. Put $x_{0}=\sum_{n=1}^{\infty} e_{n} \otimes e_{n}$ in $\left(l^{\infty}\right)^{* * *}$ $\bar{\otimes}\left(l^{\infty}\right)^{* *}$. We assert that $x_{0} \in F_{\omega}\left(l^{\infty}, l^{\infty}\right) \sim l^{\infty} \underset{\alpha}{\otimes} l^{\infty}$. Thus, from the proof of Lemma 2.2 we see that

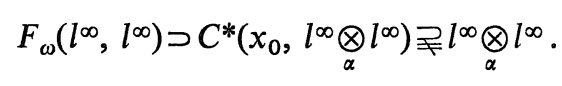

Let $\varphi$ be a singular functional of $l^{\infty}$, then $\left\langle e_{n}, \varphi\right\rangle=0$ because it is a finite linear combination of singular states. Now the right Fubini mapping for $\varphi \in\left(l^{\infty}\right)^{*}$ is a $\sigma$-weakly continuous mapping of $\left(l^{\infty}\right)^{* * \bar{\otimes}}$ $\left(l^{\infty}\right)^{* *}$ to $\left(l^{\infty}\right)^{* *}$, hence $R_{\varphi}\left(x_{0}\right)=\sum_{n=1}^{\infty}\left\langle e_{n}, \varphi\right\rangle e_{n}$ in $\left(l^{\infty}\right)^{* *}$. Therefore if $\varphi$ is singular, $R_{\varphi}\left(x_{0}\right)=0$. If $\varphi$ is normal, then $\left\langle e_{n}, \varphi\right\rangle \rightarrow 0$. Hence, $R_{\varphi}\left(x_{0}\right)$ is a uniform limit of $\sum_{i=1}^{n}\left\langle e_{i}, \varphi\right\rangle e_{i}$ and $R_{\varphi}\left(x_{0}\right) \in l^{\infty}$. We have the same conclusions for $L_{\psi}\left(x_{0}\right)$. Thus, the element $x_{0}$ belongs to $F_{\omega}\left(l^{\infty}, l^{\infty}\right)$. Next, let $\varphi_{n}$ be a normal state on $l^{\infty}$ such that $\left\langle e_{n}, \varphi_{n}\right\rangle$ $=\left\|\varphi_{n}\right\|=1$. Choose a subsequence $\left\{\varphi_{n_{i}}\right\}$ which converges to singular state $\varphi$ in the weak ${ }^{*}$ topology. Then the double sequence $\left\{\varphi_{n_{i}} \otimes \varphi_{n_{j}}\right\}$ converges in the weak* topology on $l^{\infty} \otimes l^{\infty}$. Hence for each positive number $\varepsilon$ we can find an integer $n_{\varepsilon}$ such that

$$
\left|\left\langle x_{0}, \varphi_{n_{i}} \otimes \varphi_{n_{j}}\right\rangle-\left\langle x_{0}, \varphi \otimes \varphi\right\rangle\right|<\varepsilon
$$

for every $n_{i}, n_{j}>n_{\varepsilon}$ if $x_{0}$ belonged to $l^{\infty} \underset{\alpha}{\otimes} l^{\infty}$. However this contradicts the equality;

$$
\left\langle x_{0}, \varphi_{n_{i}} \otimes \varphi_{n_{j}}\right\rangle=\sum_{n=1}^{\infty}\left\langle e_{n}, \varphi_{n_{i}}\right\rangle\left\langle e_{n}, \varphi_{n_{j}}\right\rangle=\delta_{n_{j}}^{n_{i}}
$$

Thus, the element $x_{0}$ does not belong to $l^{\infty} \underset{\alpha}{\otimes} l^{\infty}$.

Let $M$ and $N$ be von Neumann algebras. Fix an element $x \in$ 
$M \bar{\otimes} N$. For an arbitrary bounded linear functional $\varphi$ we define a bounded linear functional on $N_{*}$ as

$$
f_{x, \varphi}(\psi)=\left\langle L_{\psi}(x), \varphi\right\rangle
$$

Then there exists an element $R_{\varphi}(x)$ in $N$ such that

$$
f_{x, \varphi}(\psi)=\left\langle R_{\varphi}(x), \psi\right\rangle \text {. }
$$

We get a bounded linear mapping $R_{\varphi}$ of $M \bar{\otimes} N$ to $N$, which is clearly an extension of the right Fubini mapping in $M \otimes N$ induced by $\varphi$. We call this a generalized right Fubini mapping in $M \bar{\otimes} N$. Similarly we can define a generalized left Fubini mapping $L_{\psi}$ for any functional $\psi \in N^{*}$. From the definition we have the Fubini type principle,

$$
\left\langle R_{\varphi}(x), \psi\right\rangle=\left\langle L_{\psi}(x), \varphi\right\rangle
$$

if either $\varphi$ or $\psi$ is a normal functional. Note that the property $a R_{\varphi}(x) b$ $=R_{\varphi}(1 \otimes a x 1 \otimes b)$ holds for this generalized mapping. In fact, for $\psi \in N_{*}$,

$$
\begin{aligned}
& \left\langle a R_{\varphi}(x) b, \psi\right\rangle=\left\langle R_{\varphi}(x), R_{b} L_{a} \psi\right\rangle \\
& =\left\langle L_{R_{b} L_{a} \psi}(x), \varphi\right\rangle \\
& =\left\langle L_{\psi}(1 \otimes a x 1 \otimes b), \varphi\right\rangle=\left\langle R_{\varphi}(1 \otimes a x 1 \otimes b), \psi\right\rangle .
\end{aligned}
$$

Here the third equality follows from the property of the $\sigma$-weakly continuous mapping $L_{\psi}$.

We shall show that the $C^{*}$-algebra, $C^{*}\left(x_{0}, l^{\infty} \underset{\alpha}{\otimes} l^{\infty}\right)$ is contained in the stronger Fubini product, $F_{s}\left(l^{\infty}, l^{\infty}\right)=\left\{x \in\left(l^{\infty}\right)^{* *} \bar{\otimes}\left(l^{\infty}\right)^{* *} ; R_{\varphi}(x) \in l^{\infty}, L_{\psi}(x) \in l^{\infty}\right.$ for every $\left.\varphi, \psi \in\left(l^{\infty}\right)^{* * *}\right\}$. In fact, it is enough to show the assertion for a singular functional $\varphi$ of $\left(l^{\infty}\right)^{* *}$. We claim that $R_{\varphi}\left(x_{0}\right)=0$. Since $e_{n}$ is also a minimal projection in $\left(l^{\infty}\right)^{* *}, \varphi$ vanishes at $e_{n}$ for every $n$. Hence, putting $z_{0}=\sum_{n=1}^{\infty} e_{n}$ in $\left(l^{\infty}\right)^{* *}$, we have

$$
R_{\varphi}\left(x_{0}\right) z_{0}=\sum_{n=1}^{\infty} R_{\varphi}\left(x_{0}\right) e_{n}=\sum_{n=1}^{\infty} R_{\varphi}\left(x_{0} 1 \otimes e_{n}\right)=\sum_{n=1}^{\infty}\left\langle e_{n}, \varphi\right\rangle e_{n}=0
$$

Furthermore, 


$$
R_{\varphi}\left(x_{0}\right)\left(1-z_{0}\right)=R_{\varphi}\left(x_{0} 1 \otimes\left(1-z_{0}\right)\right)=0 \quad \text { whence } \quad R_{\varphi}\left(x_{0}\right)=0
$$

\section{§5. The Fubini Type Principle for Generalized Fubini Mappings.}

As we have seen already the Fubini type principle plays an important rôle in the theory of tensor products of operator algebras. We shall, however, prove here that we can not expect this principle to hold for our generalized Fubini mappings. Namely we have the following result, which sounds quite natural in a measure theoretical sense.

Theorem 5.1. Let $M$ be a von Neumann algebra acting on a separable Hilbert space and $N$ an infinite dimensional von Neumann algebra. Let $\varphi_{0}$ be a bounded linear functional of $M$. Then we have the equality

$$
\left\langle R_{\varphi_{0}}(x), \psi\right\rangle=\left\langle L_{\psi}(x), \varphi_{0}\right\rangle
$$

in $M \bar{\otimes} N$ for an arbitrary bounded linear functional $\psi$ of $N$ if and only if $\varphi_{0}$ is normal, i.e. $\sigma$-weakly continuous.

Proof. The sufficiency follows from the definition. So suppose $R_{\varphi_{0}}$ satisfy the above equality for any $\psi \in N^{*}$. From the assumption, the unit sphere of $M$ is metrizable in the $\sigma$-weak topology. Let $\left\{a_{n}\right\}$ be a sequence in the unit sphere of $M$ converging to zero $\sigma$-weakly. Let $\left\{e_{n}\right\}$ be an infinite family of mutually orthogonal projections in $N$. Then the von Neumann subalgebra generated by $\left\{e_{n}\right\}$ is isomorphic to the $l^{\infty}$ algebra. Write $c_{0}$ as the subalgebra of $l^{\infty}$ consisting of all sequences converging to zero. We note that $\left(l^{\infty}\right)_{*}^{\frac{1}{*}}=c_{0}^{o}$, the polar of $c_{0}$. Put $x$ $=\sum_{n=1}^{\infty} a_{n} \otimes e_{n}$ in $M \bar{\otimes} N$. If $\varphi$ is a normal functional of $M,\left\langle a_{n}, \varphi\right\rangle$ converges to 0 . Hence, $R_{\varphi}(x)=\sum_{n=1}^{\infty}\left\langle a_{n}, \varphi\right\rangle e_{n}$ belongs to $c_{0}$. Therefore if $\psi$ is an extension of a singular functional of $l^{\infty}$,

$$
\left\langle L_{\psi}(x), \varphi\right\rangle=\left\langle R_{\varphi}(x), \psi\right\rangle=0
$$

As $\varphi$ is arbitrary, it follows that $L_{\psi}(x)=0$. Hence, because of the equality, we have

$$
\left\langle R_{\varphi_{0}}(x), \psi\right\rangle=\left\langle L_{\psi}(x), \varphi_{0}\right\rangle=0
$$


which means that $R_{\varphi_{0}}(x) \in c_{0}$. Write $R_{\varphi_{0}}(x)=\sum_{n=1}^{\infty} \lambda_{n} e_{n}$ where $\lambda_{n} \rightarrow 0$. Then,

$$
\begin{aligned}
R_{\varphi_{0}}(x) e_{n}=\lambda_{n} e_{n} & =R_{\varphi_{0}}\left(x 1 \otimes e_{n}\right)=R_{\varphi_{0}}\left(a_{n} \otimes e_{n}\right) \\
& =\left\langle a_{n}, \varphi_{0}\right\rangle e_{n} .
\end{aligned}
$$

Hence, $\left\langle a_{n}, \varphi_{0}\right\rangle=\lambda_{n} \rightarrow 0$. Thus, $\varphi_{0}$ is $\sigma$-weakly continuous on the unit sphere of $M$ and by [2; Theorem 1.3.1] it is $\sigma$-weakly continuous on M.

\section{References}

[1] Akemann, C. A., Pedersen, G. K. and Tomiyama, J., Multipliers of $C^{*}$-algebras. J. Functional Analysis 13 (1973), 277-301.

[2] Dixmier, J., Les algèbıes d'opérateurs dans l'espace hilbertien, Gauthier Villars, Paris, 2nd Edition 1969.

[3] - Les $C^{*}$-algèbres et leurs représentations, Gauthier-Villars, Paris 1964.

[4] Effros, E. G., Approximation problems for $C^{*}$-algebras, preprint.

[5] Effros, E. G. and Lance, E. C., Tensor products of operator algebras, preprint.

[6] Enflo, P., A counterexample to the approximation problem in Banach spaces, Acta Math., 130 (1973), 309-317.

[7] Fell, J. M. G., The structure of operator fields, Acta Math., 106 (1961), 233280.

[8] Grothendieck, A., Produits tensoriels topologiques et espaces nucléaires, Mem. Amer. Math. Soc. (1966).

[9] Kaplansky, I., Normed algebras, Duke Math. J., 16 (1949), 399-418.

[10] Milne, H., Banach space properties of uniform algebras, Bull. London Math. Soc., 12 (1972), 323-326.

[11] Tomiyama, J. and Takesaki, M., Applications of fibre bundles to a certain class of $C^{*}$-algebras, Tôhoku Math. J., 13 (1961), 498-522.

[12] Takesaki, M., On the conjugate space of operator algebra, Tôhoku Math. J., 10 (1958), 194-203.

[13] - Singularity of positive functionals, Proc. Japan Acad., 35 (1959), 365-366.

[14] Tomiyama, J., On the product projection of norm one in the direct product of operator algebras, Tôhoku Math. J., $\mathbb{1 1}$ (1959), 303-313.

[15] Tomiyama, J., Topological representations of $C^{*}$-algebras, Tôhoku Math. J., 14 (1962), 187-204.

[16] - Applications of Fubini type theorem to the tensor products of $C^{*}$ algebras, Tôhoku Math. J., 19 (1967), 231-226.

[17] - Tensor products and projections of norm one in von Neumann algebras, Seminar at Univ. of Copenhagen 1970.

[18] - Applications of Fubini mappings to tensor products of Banach algebras, Seminar at Univ. of Copenhagen 1971.

[19] Waelbroeck, L., Duality and the injective tensor products, Math. Ann., 163 (1966), 122-126. 
